\section{Myelodysplasia-related acute myeloid leukemia and acute promyelocytic leukemia: con- comitant occurrence of two molecularly distinct diseases}

\author{
Yenny Alejandra Moreno Vanegas, ${ }^{1}$ \\ Abdel-Ghani M. Azzouqa, ${ }^{1}$ \\ David M. Menke, ${ }^{2}$ James M. Foran, ${ }^{1}$ \\ Prakash Vishnu ${ }^{1}$ \\ ${ }^{1}$ Divisions of Hematology and Medical \\ Oncology; ${ }^{2}$ Department of Laboratory \\ Medicine and Pathology, Medical \\ Oncology, Mayo Clinic, Jacksonville, \\ FL, USA
}

\begin{abstract}
Concurrent presentation of acute promyelocytic leukemia (APL) with other hematologic diseases in the absence of previous chemotherapy or ionizing radiotherapy treatment is very rare. We present a case of simultaneous occurrence of APL with myelodysplastic syndrome (MDS)-related acute myeloid leukemia (AML). A 43-yearold female presented with 3 month of history fatigue, night sweats, chills and pancytopenia. Bone marrow aspirate and biopsy demonstrated $20 \%$ myeloid blasts with dysplastic changes admixed with abnormal promyelocytes. Cytogenetic analysis showed tetraploidy and deletion in chromosomes $5 \mathrm{q}$ and $7 \mathrm{q}$ and polymerase chain reaction showed presence of PML/RARA mRNA transcripts, confirming the presence of concurrent APL and MDS-related AML. Induction chemotherapy with cytarabine and daunorubicin was initiated along with all-trans retinoic acid. This is the first case to be reported in the literature of concurrent occurrence of APL with MDS-related AML. Treatment with $7+3$ regimen and ATRA was successful in inducing complete remission.
\end{abstract}

\section{Introduction}

Myelodysplastic syndromes (MDS) represent the most common category of acquired bone marrow failure syndrome in adults. ${ }^{1}$ They are a diverse group of clonal bone marrow disorders of hematopoietic stem cells characterized by ineffective hematopoiesis, dysplasia in one or more of the major myeloid cell lines, peripheral cytopenias and increased risk of development of acute myeloid leukemia (AML). ${ }^{2}$ Failure of cellular differentiation is associ- ated with evolution to secondary AML. ${ }^{1}$ AML, a very heterogenous disease clinically, morphologically and genetically, is divided into different subtypes; one such subtype is acute promyelocytic leukemia (APL) that is characterized by the fusion of promyelocytic leukemia $(P M L)$ gene on chromosome 15 with the retinoic acid receptor alpha $(R A R \alpha)$ gene on chromosome 17.2 As a consequence, the PMLRARA oncoprotein disrupts the interaction of retinoic acid and RARA causing the arrest in maturation of hematopoietic progenitors at the promyelocyte stage. ${ }^{2}$ APL comprises about $10 \%-15 \%$ of all cases of adult AML and represents the most curable type. ${ }^{3,4}$ APL usually manifests de novo, but there are few reports of its concomitant occurrence with chronic lymphocytic leukemia (CLL) and mantle cell lymphoma (MCL), mainly attributed to previous use of chemotherapy and/or radiotherapy. 5,6 Concurrent occurrence of APL with other hematologic diseases in the absence of therapy is even rarer; there are few anecdotal reports of plasma cell myeloma and myeloproliferative neoplasms co-existing with APL in the absence of therapy. ${ }^{7,8}$ Herein, we present a case of concomitant occurrence of APL with MDS-related AML.

\section{Case Report}

A 43-year-old female of Caucasian ancestry was evaluated in hematology clinic for symptoms of progressive fatigue, generalized bone pains, and night sweats of 3 months duration, and a complete blood count obtained by her primary care physician showing pancytopenia with white blood cell count of $1.5 \times 10^{3} / \mu \mathrm{L}$, absolute neutrophil count of $0.8 \times 10^{3} / \mu \mathrm{L}$, hemoglobin of $9.8 \mathrm{~g} / \mathrm{dL}$ with an MCV $99 \mathrm{fL}$, and a platelet count of $116 \times 10^{3} / \mu \mathrm{L}$. Her past medical history was significant for eosinophilic esophagitis and generalized anxiety disorder. She did not have any history of tobacco, alcohol or other substance use, and no history of exposure to chemotherapy or ionizing radiation. There wasn't any family history of primary hematologic disorders. On examination, the patient appeared well, without any signs of mucocutaneous bleeding or splenomegaly.

Peripheral blood film showed anisocytosis and polychromasia in the red cell lineage but without any noticeable morphologic abnormalities in the leukocytes or platelets. Bone marrow biopsy was hypercellular with a cellularity-to-fat ratio of 80:20. The myeloid blasts were increased estimated to be $20 \%$ with admixed abnormal promyelo-
Correspondence: Prakash Vishnu, Division of Hematology and Medical Oncology, Mayo Clinic, 4500 San Pablo Road, Jacksonville, FL 32224, USA.

Tel.: +1.904.953.2693 - Fax: +1.904.953.0984.

E-mail: vishnu.prakash@mayo.edu

Key words: Concurrent; acute myeloid leukemia; myelodysplastic syndrome; acute promyelocytic leukemia; management.

Contributions: the authors contributed equally.

Conflicts of interest: the authors declare no potential conflict of interest.

Received for publication: 26 February 2018. Accepted for publication: 24 June 2018.

This work is licensed under a Creative Commons Attribution-NonCommercial 4.0 International License (CC BY-NC 4.0).

CCopyright Y.A.M. Vanegas et al., 2018

Licensee PAGEPress, Italy

Hematology Reports 2018; 10:7658

doi:10.4081/hr.2018.7658

cytes $(20 \%)$. There was myelofibrosis (grade 2+), dysmyelopoietic changes with pseudo Pelger-Huet cells, decreased erythropoiesis and abnormal megakaryocytes (Figure 1). Cytogenetic analysis by Giemsa banding showed tetraploidy and deletion in chromosomes $5 \mathrm{q}$ and $7 \mathrm{q}[92, \mathrm{XXXX}$, del (5)(q22q35), del (7)(q22q34)] in 6 of the 20 metaphases.

AML fluorescence in situ hybridization (FISH) showed that $12 \%$ of nuclei were tetraploid, however there was no fusion of the PML and RARA gene regions or the MYH11 and CBFB gene regions. Quantitative real-time polymerase chain reaction (qRT-PCR) detected PML/RARA mRNA transcripts, estimated to be $31.3 \%$ (normalized value of PML-RARA to GUSB control gene transcripts), indicating the presence of acute promyelocytic leukemia (APL) clone. OncoHeme next-generation sequencing (https://www.mayomedicallabo r a t o r i e s. c o m/t e s t catalog/Overview/63367) did not show any pathogenic mutations. The diagnosis was consistent with co-occurrence of APL and MDS-related AML. The patient was admitted for induction chemotherapy with $7+3$ regimen (cytarabine $125 \mathrm{mg} / \mathrm{m}^{2} /$ day for 7 days and daunorubicin $90 \mathrm{mg} / \mathrm{m}^{2} /$ day for 3 days) and all-trans retinoic acid (ATRA) 45 $\mathrm{mg} / \mathrm{m}^{2} /$ day. Post-induction bone marrow biopsy obtained in day 14 presented with residual leukemic blasts but without any mRNA PML/RARA transcripts. She hence underwent second induction with $5+2$ reg- 
imen (cytarabine $100 \mathrm{mg} / \mathrm{m}^{2} /$ day for 5 days and daunorubicin $50 \mathrm{mg} / \mathrm{m}^{2} /$ day for 2 days). A repeat bone marrow biopsy obtained once peripheral blood cell counts normalized showed both a morphologic and molecular remission. She subsequently received consolidation chemotherapy with 2 cycles of high dose cytarabine (HiDAC, $3 \mathrm{~g} / \mathrm{m}^{2}$ q. 12 hours on days 1, 3 and 5). Search for a suitable donor for allogeneic hematopoietic stem cell transplant is underway.

\section{Discussion}

We present the first case report of a patient with concomitant occurrence of APL and MDS-related AML, and its management. Although reports of concurrent APL and other hematological disease do exist, they are very rare and are usually therapy-related. Lim et al. in 2014 described a 60 year old male with APL concomitant with plasma cell myeloma without previous exposure to chemotherapy and radiotherapy. ${ }^{7}$ They proposed that the concurrent presentation of these two entities was a result of abnormal multipotent stem cells, environmental exposure that gave rise to the leukemic clone. ${ }^{7}$ The patient was treated with ATRA; idarubicin was not administered due to sepsis. ${ }^{7}$ Su et al. in 2017 reported a case of a 52 year old male with chronic lymphocytic leukemia (CLL) and APL treated with ATRA and hydroxurea. ${ }^{9}$ The patient developed acute hypoxic respiratory failure and did not respond to treatment. $\mathrm{He}$ developed disseminated intravascular coagulation and eventually died. MamorskaDyga et al. in 2016 described a young patient with co-existing APL with $J A K 2$ $V 617 F$ positive myeloproliferative neoplasm (MPN). ${ }^{8}$ The patient was induced with ATRA and arsenic trioxide (ATO) and developed thrombocytosis with a bone marrow biopsy that showed reticulin fibrosis, left shift of myeloid lineage and dysplastic changes. ${ }^{8}$ The patient was refractory to ATRA and ATO treatment and was treated with cytarabine and idarubicin, and eventually with hydroxyurea and low dose aspirin. ${ }^{8}$ They hypothesize that the JAK2 $V 617 F$ positive MPN clone was present first and that additional mutations on FLT3 and $P M L / R A R A$ led to the development of APL. ${ }^{8}$ Akin to many cases, the etiology for MDS-related AML in our patient is not clear. The chromosomal abnormalities found in our patient (tetraploidy, del $5 \mathrm{q}$ and del 7q) can occur de novo but are more commonly present in therapy-related AML/MDS (tAML/tMDS). ${ }^{2,10}[11]$ The occurrence of therapy related AML as a complication of cytotoxic therapy is well documented and has a poor prognosis. ${ }^{2} \mathrm{~A}$ study by Duffield et al. demonstrated that

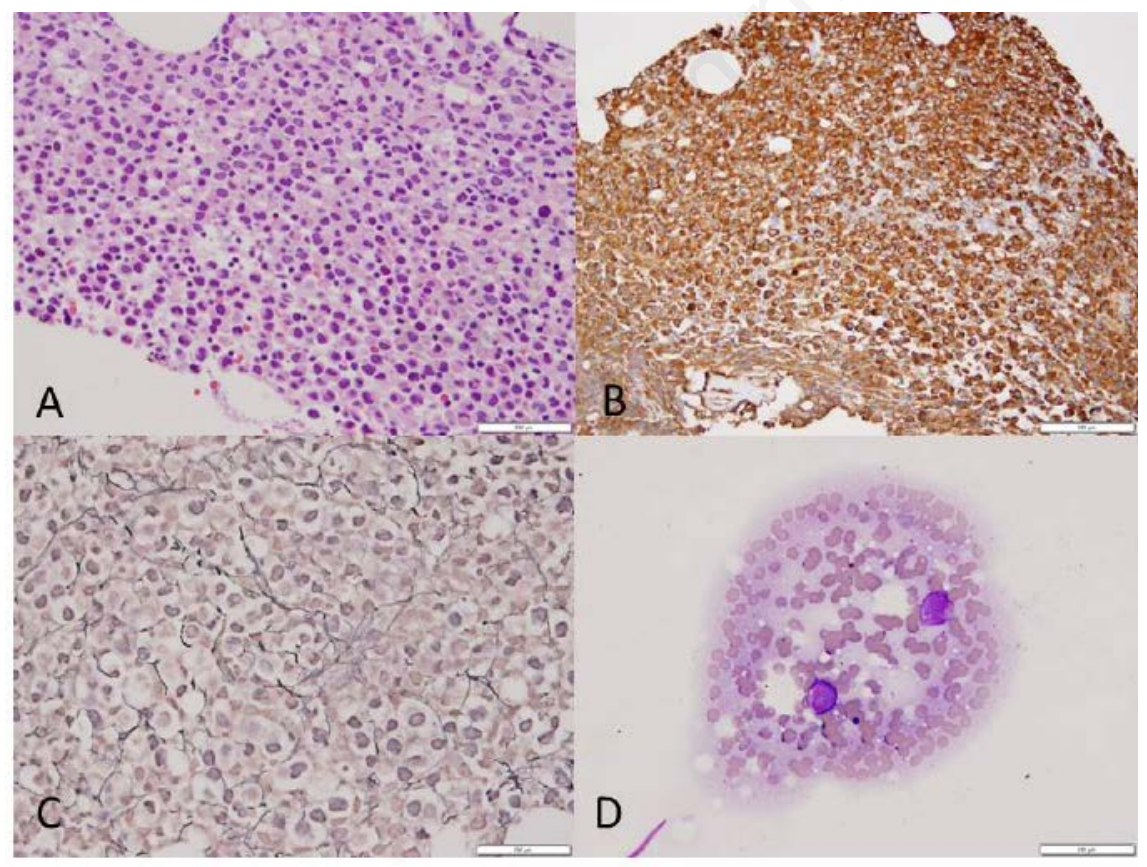

Figure 1. Bone marrow biopsy pathology slides with different stains. A) Bone marrow biopsy 100X hematoxylin and eosin stain showing a hypercellular marrow with increased promyelocytes; B) Bone marrow biopsy 200X MPO stain showing diffuse MPO staining; C) Bone Marrow Biopsy 400X reticulin stain showing grade 1 fibrosis; D) Roll preparation 400X Wright stain showing promyelocyte blasts. about $9 \%$ of APL cases in their cohort presented after treatment with cytotoxic chemotherapy or radiation therapy. ${ }^{11}$ Our patient did not have any past medical history of any other malignant diseases, or prior use of chemotherapy or ionizing radiation. The most common genetic abnormality in APL is the translocation between chromosome 15 and 17 [t(15;17)(q22; 12$)]$ but different translocation partners of RARA gene have been described with clinical and pathological findings similar to APL. These include $\mathrm{t}(11 ; 17)(\mathrm{q} 23 ; \mathrm{q} 21)$ ZBTB16/RARA, $\mathrm{t}(11 ; 17)(\mathrm{q} 13 ; \mathrm{q} 21) \quad N U M A / R A R A$, $\mathrm{t}(5 ; 17)(\mathrm{q} 35 ; \mathrm{q} 21) \quad N P M / R A R A, \quad \operatorname{der}(17)$ STAT5B/RARA, $\operatorname{der(17)~PRKARla/RARA,~}$ $\mathrm{t}(\mathrm{X} ; 17)(\mathrm{p} 11 ; \mathrm{q} 12) \quad B C O R / R A R A$, and $\mathrm{t}(4 ; 17)(\mathrm{q} 12 ; \mathrm{q} 21)$ FIP1L1/RARA. ${ }^{12}$ These findings are indicative of the key role of $R A R A$ in the pathogenesis of the disease. ${ }^{2}$ We suspect that the $P M L / R A R A$ translocation is an independent event, causing the distinct APL clone.

The mainstay of treatment for MDSrelated AML is induction chemotherapy with anthracycline and cytarabine, and the best chance at cure is consolidation with allogenic hematopoietic stem cell transplant if the patient is deemed appropriate candidate and if there is availability of a suitable donor. $^{13-15}$ Long term survival rates in patients with first complete remission are often higher than $50 \% .{ }^{15}$ Since our patient was relatively young and did not have any major comorbidities, induction chemotherapy with $7+3$ regimen was deemed the most appropriate treatment. On the other hand, the standard treatment for de novo APL is anthracyclines and ATRA, which induces terminal differentiation followed by natural apoptosis of malignant cells. ${ }^{16}$ The combination of anthracyclines and ATRA induces a complete response in over $90 \%$ of patients, and the risk of relapse is $16.5 \%$ at 10 years. ${ }^{17}$ The main side effects associated with ATRA include the differentiation syndrome which manifests with fever, respiratory distress, weight gain, pleural or pericardial effusions and sometimes renal failure. ${ }^{16}$ Other side effects from prolonged use of ATRA include Sweet syndrome which is an acute febrile neutrophilic dermatosis with fever, leukocytosis, raised plaques on the skin and histologically dense, dermal infiltration of the skin with mature neutrophils. ${ }^{18}$

In our patient, the combination treatment was successful in inducing remission of both MDS-related AML and APL. She began consolidation therapy with HiDAC. 


\section{Conclusions}

This is the first case report of concurrent occurrence of APL and MDS-related AML, and the description of its management. Treatment with combination of $7+3$ regimen and ATRA was successful in inducing a complete remission from both APL and MDS-related AML. The optimal consolidation therapy in such cases where there are 2 clones of AML, remains unknown. Our patient received consolidation chemotherapy with 2 cycles of HiDAC, and search for a suitable donor for allogenic hematopoietic stem cell transplant is underway.

\section{References}

1. Bejar R, Stevenson KE, Caughey B, et al. Somatic mutations predict poor outcome in patients with myelodysplastic syndrome after hematopoietic stem-cell transplantation. J Clin Oncol 2014;32: 2691-8.

2. Swerdlow S, Campo E, Harris N, et al. WHO classification of tumours and haematopoietic and lymphoid tissues. Lyon: IARC; 2008.

3. Hoffman R. Hematology: basic principles and practice. Philadelphia, PA: Saunders/Elsevier; 2013. Available from: https://www.clinicalkey. com/dura/browse/bookChapter/3-s2.0C20100668751

4. Naini AE, Karbalaie A, Abedini M, et al. Comparison of malnutrition in hemodialysis and peritoneal dialysis patients and its relationship with echocardiographic findings. J Res Med Sci 2016;21:78.

5. Molero T, Lemes A, de la Iglesia S, et al. Acute promyelocytic leukemia developing after radiotherapy for prostate cancer in a patient with chronic lymphocytic leukemia. Cancer Genet Cytogenet 2001;131:141-3.

6. Au WY, Chim CS, Wai Lie AK, et al. Real-time quantification of the multidrug resistance-1 gene expression in relapsed acute promyelocytic leukemia treated with arsenic trioxide. Haematologica 2002;87:1109-11.

7. Lim J, Kwon GC, Koo SH, et al. A case of acute promyelocytic leukemia concomitant with plasma cell myeloma. Ann Lab Med 2014;34:152-4.

8. Mamorska-Dyga A, Wu J, Khattar P, et al. Acute promyelocytic leukemia coexisting with JAK2 V617F positive myeloproliferative neoplasm: a case report. Stem Cell Investig 2016;3:8.

9. Su J, Veillon D, Shackelford R, et al. Acute promyelocytic leukemia and chronic lymphocytic leukemia: concomitant presentation of two molecularly distinct entities. J La State Med Soc 2017;169:68-70.

10. Arber DA, Orazi A, Hasserjian R, et al. The 2016 revision to the World Health Organization classification of myeloid neoplasms and acute leukemia. Blood 2016;127:2391-405.

11. Duffield AS, Aoki J, Levis M, et al. Clinical and pathologic features of sec- ondary acute promyelocytic leukemia. Am J Clin Pathol 2012;137:395-402.

12. Singh MK, Parihar M, Arora N, et al. Diagnosis of variant RARA translocation using standard dual-color dualfusion PML/RARA FISH probes: An illustrative report. Hematol Oncol Stem Cell Ther 2017 [Epub ahead of print].

13. Garcia-Manero G. Integrating care for patients with lower risk myelodysplastic syndrome. Semin Oncol 2011;38:658-66.

14. Ostgard LS, Kjeldsen E, Holm MS, et al. Reasons for treating secondary AML as de novo AML. Eur J Haematol 2010;85:217-26.

15. Phillips GL. Allogeneic hematopoietic stem cell transplantation (HSCT) for high-risk acute myeloid leukemia (AML)/myelodysplastic syndrome (MDS): how can we improve outcomes in the near future? Leuk Res 2012;36:1490-5.

16. Cassinat B, Chevret S, Zassadowski F, et al. In vitro all-trans retinoic acid sensitivity of acute promyelocytic leukemia blasts: a novel indicator of poor patient outcome. Blood 2001;98:2862-4.

17. Ades L, Guerci A, Raffoux E, et al. Very long-term outcome of acute promyelocytic leukemia after treatment with alltrans retinoic acid and chemotherapy: the European APL Group experience. Blood 2010;115:1690-6.

18. Thompson DF, Montarella KE. Druginduced Sweet's syndrome. Ann Pharmacother 2007;41:802-11. 